\title{
SII-2a
}

\section{Optical simulation of a parabolic solar concentrator}

\author{
Serge Dzo Mawuefa AFENYIVEH ${ }^{1}$, Assiongbon ADANLETE ADJANOH ${ }^{1}$, Dam-Be Lardja DOUTI $^{1}$
}

\section{Abstract}

This work presents a 3D ray tracing approach for the optical simulation of a parabolic solar concentrator for a thermo-magnetic (Curie motor) experiment. An approach based on Snell's law of reflection (geometrical optics) in vector form is used in this study. We consider the solid angle of the sun through a rotation of the concentrator around an axis. This paper aims to set up a digital technical tool as flexible as possible for applications in the field of renewable energy. Mathematical modelling as well as numerical simulation in Python environment are presented. The aim of the proposed algorithm is to be used for the concentration of light rays on any optical system, symmetrical or not. Better yet, offers a solar tracking model.

Keywords: solar parabolic concentrator, optical modelling, ray tracing simulation

\section{Introduction}

The issue of energy availability is of great interest to the scientific community. As an alternative to this concern, the development of renewable energies has become necessary [1]. This is how sources of renewable energy are included in the list of solutions to the critical situation of the energetic crisis that the world is facing. Indeed, the exploitation of these sources not only reduces the dependence on traditional resources but also preserve the environment. Thermodynamic solar transforms solar energy into heat at high temperature, then converts this heat into electrical energy. Several thermodynamic conversion technologies are presented in the literature (parabolic trough systems, solar tower systems, solar dish systems and linear Fresnel system) [2]. However, the performance of the system depends on several parameters. The parabolic solar concentrator system offers the possibility of producing heat from solar energy, temperatures can easily exceed $800^{\circ} \mathrm{C}$ and the conversion efficiency is generally high [3]. By the way, since the sun's disc has a finite angular size of $0.53^{\circ}$, light rays reaching the earth surface are not really parallel. Therefore, instead of the incident rays being reflected on the focal point, the reflected rays form an image centered at the optical focus $[4,5,6]$. The image formed on the focus can be analyzed using a ray tracer [7]. Indeed, the ray tracing is a technique that tracks rays from the source to the final point [8]. It consists of tracing the individual trajectories of the solar rays passing through the optical system and their spatial distribution. This technique has the ability to handle any kind of geometry with a great precision. The ray tracing model is then important for the design of a suitable geometry of ray concentrator. This study aims to develop a homemade calculation code as general as possible for our laboratory, for the concentration of solar radiation and which can follow the solar axis. The work is presented in 3D system and the vector formulation of Snell's law of reflection [9] is used to represent the reflected rays.

\footnotetext{
${ }^{1}$ Laboratoire Matériaux, Energie Renouvelable et Environnement (LaMERE), Université de Kara
} 


\section{Theoretical description}

We describe here the analytical concepts and present the mathematical approach which will be subsequently solved under Python.

\subsection{Geometrical aspects}

Let's first of all determine the 3D parabolic surface according to the axe and angle of rotation. The general equation of paraboloids in $(O, X, Y, Z)$ coordinate system is given by:

$$
\left(E_{\text {surf }}^{0}\right): Z=\alpha X^{2}+\beta Y^{2}
$$

The rotation of a vector $\vec{V}$ by an angle $\psi$ around a unit vector $\vec{u}$ can be described by a transfer matrix $R_{u}^{\psi}$; so that the coordinate of the new vector $\overrightarrow{V^{\prime}}$ is define by the relation:

with

$$
\overrightarrow{V^{\prime}}=R_{u}^{\psi} \cdot \vec{V}
$$

$$
R_{u}^{\psi}=\left[\begin{array}{ccc}
u_{x}^{2}(1-\cos \psi)+\cos \psi & u_{x} u_{y}(1-\cos \psi)-u_{z} \sin \psi & u_{x} u_{z}(1-\cos \psi)+u_{y} \sin \psi \\
u_{x} u_{y}(1-\cos \psi)+u_{x} \sin \psi & u_{y}^{2}(1-\cos \psi)+\cos \psi & u_{y} u_{z}(1-\cos \psi)-u_{x} \sin \psi \\
u_{x} u_{z}(1-\cos \psi)-u_{y} \sin \psi & u_{y} u_{z}(1-\cos \psi)+u_{x} \sin \psi & u_{z}^{2}(1-\sin \psi)+\cos \psi
\end{array}\right]
$$

For a rotation around the $(O X)$ axis, $\vec{u}=(1,0,0)$, the transfer matrix $R_{u}^{\psi}$ can be expressed as:

$$
R_{O X}^{\psi}=\left[\begin{array}{ccc}
1 & 0 & 0 \\
0 & \cos \psi & -\sin \psi \\
0 & \sin \psi & \cos \psi
\end{array}\right]
$$

The general equation of the paraboloid is then given by:

$$
E_{\text {surf }}^{\psi}=R_{u}^{\psi} * E_{\text {surf }}^{0}
$$

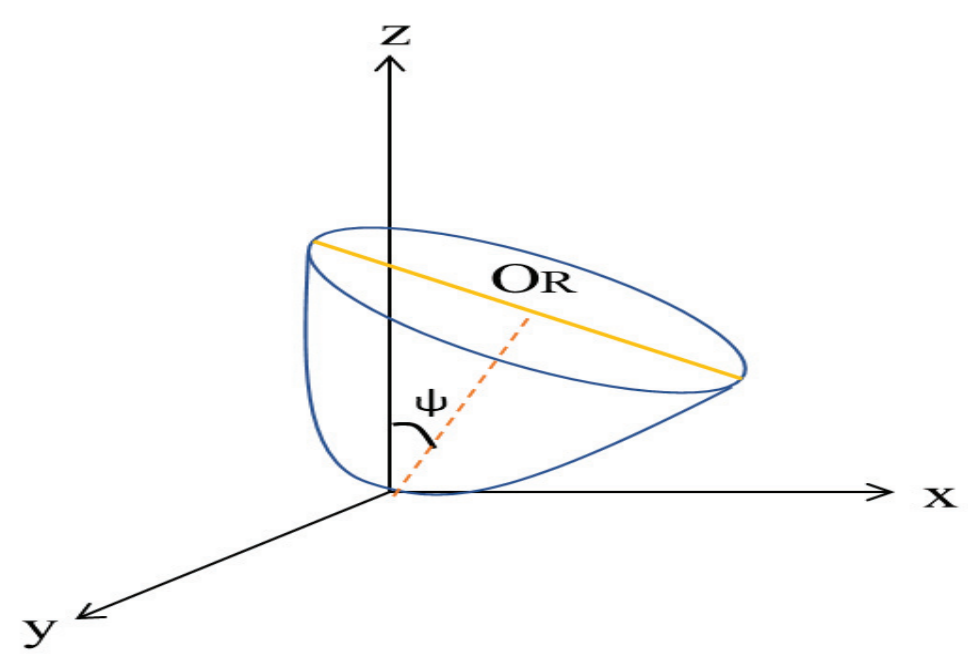

Figure 1: Parabolic surface inclined at angle $\psi$

The solar collector is characterized by its minor and major axes lengths, which will define the solid angle of the solar collection. For the optical calculations, we have to define the maximum angle of the solar rays that will be collected. The sun position is denoted by $S\left(x_{s}, y_{s}, z_{s}\right)$ and the solar rays localised using spherical coordinate angles $(\theta, \varphi)$ so that an incident ray from the sun will be characterised by its unit vector $\vec{u}_{i}$, defined by: 


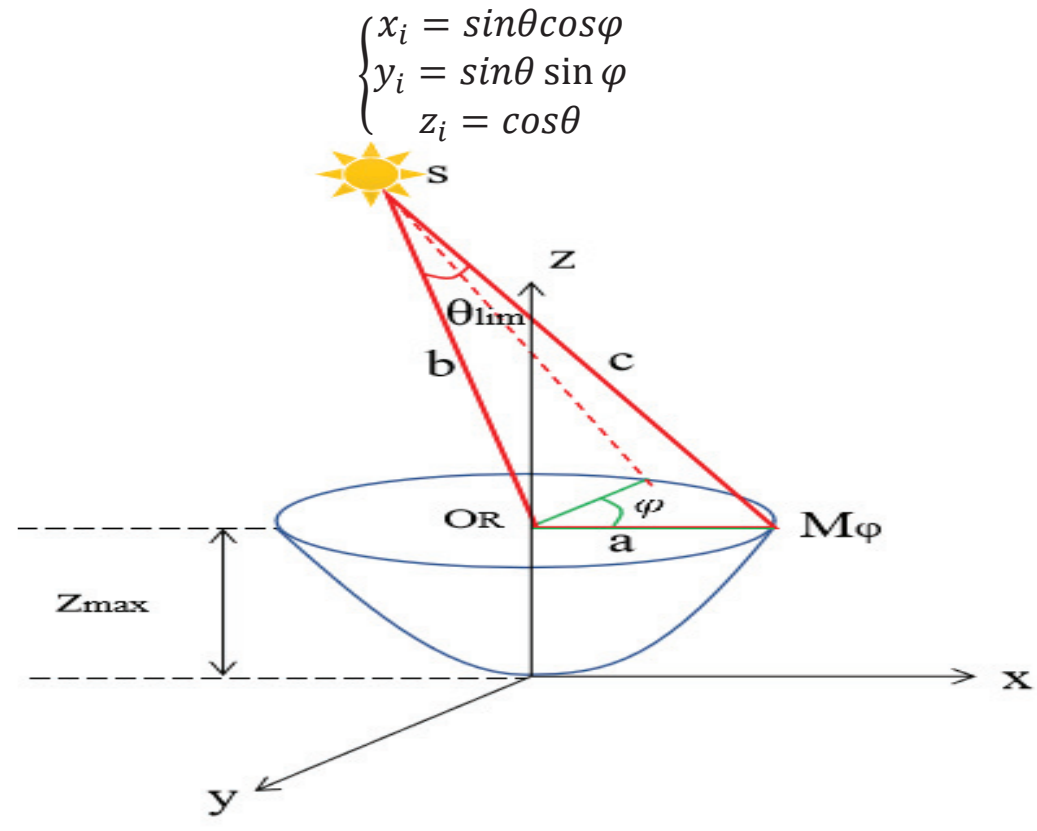

Figure 1: Geometric representation of the system

Without rotation, the aperture of the parabola is defined by an ellipse which parametric equation can be written as:

$$
\left\{\begin{array}{c}
x_{e}^{0}=\frac{\operatorname{diam}_{x}}{2} \cos \varphi \\
y_{e}^{0}=\frac{\operatorname{diam}_{y}}{2} \sin \varphi \\
z_{e}^{0}=Z \max
\end{array}\right.
$$

After rotation, the aperture equation will now be defined as:

$$
R_{O X}^{\psi} *\left(\begin{array}{c}
x_{e}^{0} \\
y_{e}^{0} \\
z_{e}^{0}
\end{array}\right)=\left(\begin{array}{l}
x_{e} \\
y_{e} \\
z_{e}
\end{array}\right)=\vec{u}_{\text {conic }}
$$

Let's call the centre of the aperture ellipse $O_{R}$, and $M \varphi$ any point on the ellipse, We have then:

$$
O_{R}=R_{O X}^{\psi}\left(\begin{array}{c}
0 \\
0 \\
\text { zmax }
\end{array}\right) \text { and } \overrightarrow{O_{R} M_{\varphi}}=\vec{u}_{\text {conic }}
$$

The limit angle, $\theta_{\text {lim }}$ of the incidence can then be deduced by Al-kashi theorem applied on the triangle $\left(S O_{R} M_{\varphi}\right)$ :

With

$$
\cos \left(\theta_{\lim }\right)=\frac{b^{2}+c^{2}-a^{2}}{2 b c}
$$

$$
\left\{\begin{array}{c}
a=\left\|\overrightarrow{O_{R} M_{\varphi}}\right\| \\
b=\left\|\overrightarrow{O_{R} S}\right\| \\
c=\left\|\overrightarrow{S M_{\varphi}}\right\|
\end{array}\right.
$$




\subsection{Optical aspect}

The interest of this section is to find the reflected ray for each incident on the solar parabolic surface on the basis of the vector formulation of Snell's reflection law.

Let $\left.\mathrm{M}\right|_{z} ^{x} y$ be a point in $3 \mathrm{D}$ space. The incident ray equation $\left(E_{i}\right)$, is define by its origin $\mathrm{S}$ (sun position) and unit vector $\overrightarrow{u_{\imath}}$ as given by the relation:

$\overrightarrow{S M}=\lambda \overrightarrow{u_{\imath}}$, which implies that $\left\{\begin{array}{l}x=x_{s}+\lambda x_{i} \\ y=y_{s}+\lambda y_{i} \\ z=z_{s}+\lambda z_{i}\end{array}\right.$

Let's now deduce the intersection point of the incident rays with the parabolic surface, by equalizing the incident ray equation $\left(E_{i}\right)$ with the parabola surface $\left(E_{\text {surf }}^{\psi}\right)$. One would have a second order equation to be solved:

$$
\left(E_{\text {inters }}\right): \mu_{1} z^{2}+\mu_{2} z+\mu_{3}=0
$$

The appropriate value of $Z_{\text {inters }}$ is expressed by:

$$
z_{\text {inter }}=\operatorname{Roots}\left\{\left[E_{\text {inters }}\right]\right\} \cap\left[0, z_{\vec{u}_{\text {conic }}}\right]
$$

The normal vector is defined by equation 13 and is computed according to $(\theta, \varphi)$

$$
\vec{n}=\frac{\vec{\nabla} E_{\text {surf }}^{\psi}}{\left\|\vec{\nabla} E_{\text {surf }}^{\psi}\right\|}
$$

Now, we have all the necessary elements to compute the reflected ray. Let's then consider equation 14, which is the vector formulation of Snell's law of reflection.

$$
\vec{u}_{r}=\vec{u}_{i}-2\left[\left(\vec{u}_{i} \cdot \vec{n}\right) \cdot \vec{n}\right]
$$

Knowing the coordinates of the intersection point $M_{\text {inters }}\left(x_{\text {inters }}, y_{\text {inters }}, z_{\text {inters }}\right)$ and the reflected rays vector orientation $\vec{u}_{r}\left(x_{r}, y_{r}, z_{r}\right)$, the parametric equation is given by:

$$
\left\{\begin{array}{l}
x=x_{\text {inters }}+\lambda x_{r} \\
y=y_{\text {inters }}+\lambda y_{r} \\
z=z_{\text {inters }}+\lambda z_{r}
\end{array}\right.
$$

\section{Simulation results}

The results of numerical simulation under Python programming language is presented here. Rays are represented considering the astronomical unit and the solid angle of the solar collector. The proposed calculation code is quite flexible. Thus, values such as the coefficients $\alpha$ and $\beta$ of the general equation of the parabolic surface as well as the $Z_{\max }$ of the concentrator and the number of rays to be represented can be varied depending on the application.

\begin{tabular}{|c|c|c|c|}
\hline $\boldsymbol{\alpha}$ & $\boldsymbol{\beta}$ & $\boldsymbol{Z}_{\max }$ & Number of rays \\
\hline 0.1 & 0.1 & 1 & $1 \mathrm{e} 4$ \\
\hline
\end{tabular}

Table 1: Input parameters of simulation 


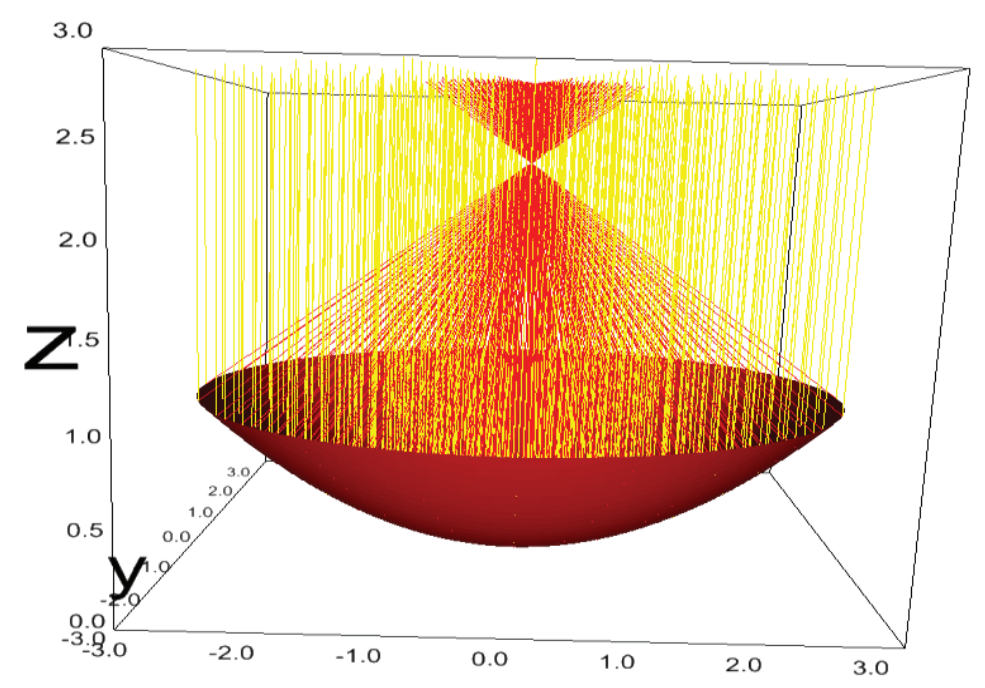

Figure 3: Representation of incident (yellow) and reflected (red) rays

Note that in reality, incident rays are not parallel as shown in the figure. Indeed, this impression comes from the fact that the source is very far from the parabolic surface. Now we can simulate the ray distribution at the focal point.

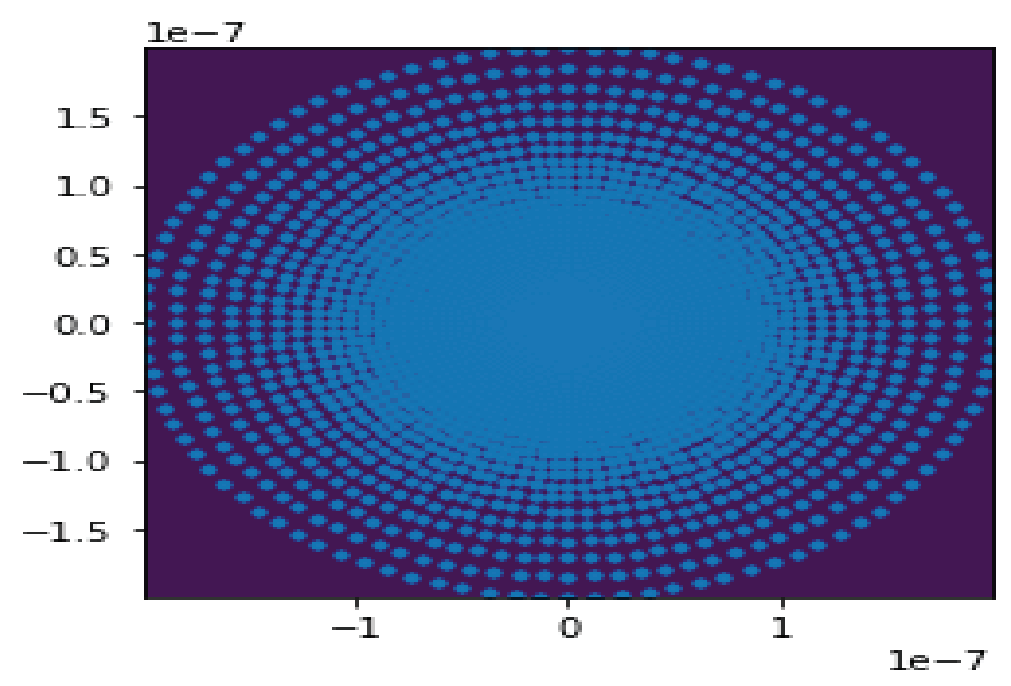

Figure 4: Solar ray distribution at focus plan (1e4 rays has been traced)

As application of the optical analysis, we simulate the energy map at the focus of our system, for a daily mean radiation of $6 \mathrm{Wh} \cdot \mathrm{m}^{-2} \cdot d^{-1}$ collected by the parabola defined in table 1 . 


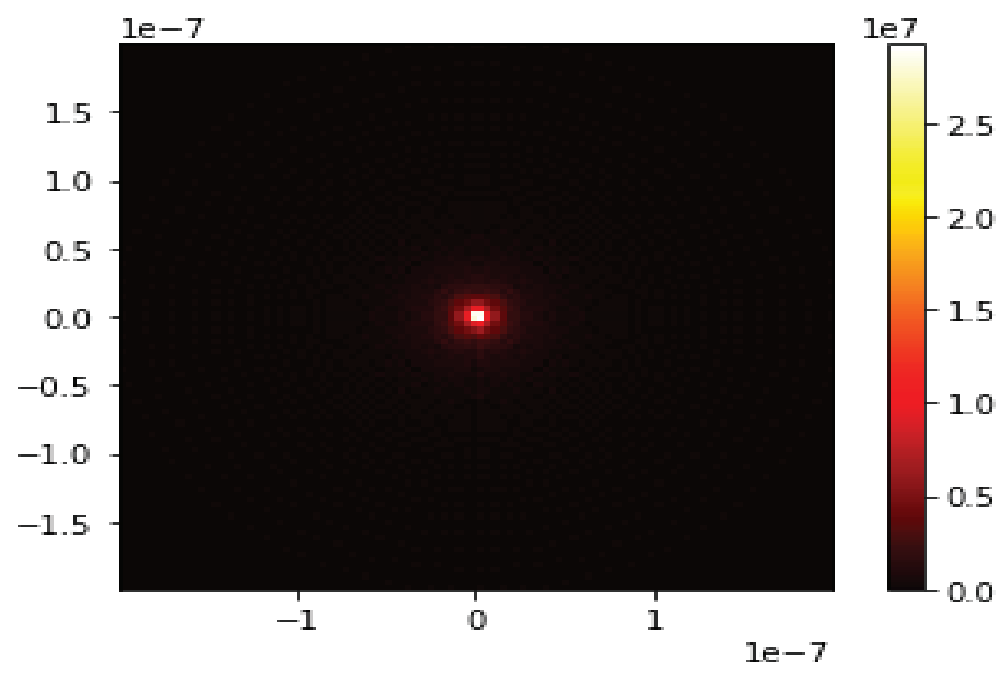

Figure 5: Spatial distribution of energy in the focal plane: the colour map values are in Joules $(\mathrm{J})$.

We can notice that the intensity of the solar ray is concentrated in the centre and is reduced as one moves away from it.

\section{Conclusion and perspectives}

This paper presents an approach of 3D rays tracing. A theorical model is proposed and an optical simulation is developed in Python environment. The quality of results obtained is satisfactory. The model can make it possible to focus all the rays reflected by a parabolic surface.

This calculation code could be used for many applications.

These results can be improved. We therefore project to:

- Finalize the simulation with the tracking system, based on the theoretically described algorithm; - Optimize the system by varying the different physical parameters in order to obtain an efficiency matrix;

- Make a thermal heat application of the concentrator;

- Simulate ferromagnetic materials in order to obtain the one with the lowest Curie temperature and which, according to the study conditions, will produce a more intense magnetic field.

\section{References}

[1] E. Al Ibrahmi, S. E Lachhab, F. Hamdaoui, I. El Amrani, A. Achhar and L. Dlimi, "Modeling of a cylindro-parabolic solar collector and simulation by Matlab software", Int. J. of Sc. \& Eng. Res., vol.8, no.10, pp.1135-1139, Oct. 2017, ISSN 2229-5518.

[2] A. Z. Hafez, A. Soliman, K. A. El-Metwally, I. M. Ismael, "Solar parabolic dish stirling engine system design, simulation and thermal analysis", Energy conversion and management, vol.126, pp.60-75, Jul.2016, doi:101016/j.enconman.2016.07.067.

[3] B. Maaoui, Y. Aoun and S. Benramache, "Réalisation d'un four solaire pour l'élaboration des oxydes métalliques", The $5^{\text {th }}$ Int. Seminar on New and Ren. En., Ghardaia-Ageria, 24-25 October 2018.

[4] A. Chikuwa and J. Lovseth, "Modeling of a novel volumetric absorber, suitable for compact solar concentrator with rock storage", Proceeding of ISES Solar World Congress, Johanesburg, 12-14 October 2009. 
[5] J. A. Dufie and W. A. Beckam, "Solar Eng. of Thermal Processes, third edition", John Wiley and Sons, Inc., Hoboken, New Jersey, 2006.

[6] A. Mobarak and A. A. Rahim, "Determination of local flux distribution of a parabolic dish solar concentrator applying real shape”, $12^{\text {th }}$ Annual Int.Solar En. Conference, ASME, pp. 7986.

[7] K. Nyenga, D. Okello and O. J. Nydal, "A ray tracer model for analysis of solar concentrating systems”, J. of En. In Southern Africa, vol.30, no.1, pp. 8-20, Feb,2019, doi:10.17159/24133051/2019/v30i1a5267.

[8] F. J. Seron, D. Gutierrez, G. Gutierrez, and E. Cerezo, "Implementation of a method of curved ray tracing for inhomogeneous atmospheres", Computers \& Graphics, vol.29, no.1, pp. 95-109, Feb. 2005, doi:10.1016/j.cag.2004.11.010.

[9] V. V. Fisanov, "Vector formulations of the laws of reflection and refraction of forward and backward waves", Russian Phys. J., vol. 58, no 8, pp. 1074-1079, Dec 2015, doi:101007/s11182-015-0614-6. 